\title{
Fish oil supplementation in a porcine model of aortic vascular prosthetic graft infection
}

\author{
S. N. Langerhuus ${ }^{1}$, B. M. Damgaard ${ }^{2}$ and C. Lauridsen ${ }^{1}$ \\ ${ }^{1}$ Research Group for Immunology and Microbiology and ${ }^{2}$ Research Group for Integrative Physiology, Department of \\ Animal Health and Bioscience, Aarhus University, P.O. Box 50, 8830 Tjele, Denmark
}

Polyunsaturated $n-3$ fatty acids in fish oil are potential anti-inflammatory agents, and may be of benefit to patients at risk of developing sepsis. They act directly by replacing arachidonic acid (AA) as an eicosanoid substrate and inhibiting AA metabolism, and indirectly, by altering inflammatory gene transcription ${ }^{(1)}$. Aortic vascular prosthetic graft infection (AVPGI) is a feared post-operative complication, and a frequently involved pathogen is Staphylococcus aureus (S. aureus). Due to lack of powerful randomised humane trials in this area, a porcine model on AVPGI was developed in order to study both nutritional and surgical strategies against this type of infection. The objective of the present study was to investigate the effect of fish oil supplementation compared to sunflower oil and animal fat supplementation on erythrocyte fatty acid composition and the concentration of the AA metabolite, prostaglandin, in plasma.

In this study, 60 pigs were randomised to three diets containing $10 \%$ fat, based on fish oil, sunflower oil or animal fat. The pigs were fed the diets for 35 days, and after 21 days all had surgery. During anaesthesia an aortic vascular prosthetic graft was inserted in the abdominal part of the aorta, and the grafts were artificially infected with $S$. aureus (strain ATCC29213, 10 ${ }^{6}$ colony forming units). The surgical development of the porcine model on AVPGI has been described elsewhere ${ }^{(2)}$. Blood samples were drawn at three time points; start of trial (day 0), just prior to AVPGI (day 21) and at end of trial (day 35). The content of AA and eicosapentaenoic acid (EPA) in erythrocytes were analysed by gas chromatography. A commercial Elisa kit (Caymann Chemical Company, Ann Arbor, USA) was used to determine plasma $\mathrm{PGE}_{2}$ metabolite concentration (13,14-dihydro-15-keto-metabolite). Results are given in the table below as slopes ( $\beta$ ) with $95 \%$ confidence intervals.

Changes in erythrocyte fatty acid content $(\%)$ and plasma $\mathrm{PGE}_{2}$ metabolite concentration (pg/ml) per day

\begin{tabular}{llclr}
\hline Supplementation & & Fish oil $(n=20)$ & Sunflower oil $(n=20)$ & Animal fat $(n=20)$ \\
\hline AA $(\% / d)$ & $\beta$ & $-0.07(-0.08 ;-0.07)^{\mathrm{a}}$ & $-0.01(-0.004 ;-0.02)^{\mathrm{b}}$ & $0.004(-0.004 ; 0.01)^{\mathrm{c}}$ \\
EPA $(\% / \mathrm{d})$ & $\beta$ & $0.16(0.14 ; 0.17)^{\mathrm{a}}$ & $-0.005(-0.02 ; 0.01)^{\mathrm{b}}$ & $-0.002(-0.02 ; 0.01)^{\mathrm{b}}$ \\
PGE $_{2}$ metabolite $(\mathrm{pg} / \mathrm{ml} / \mathrm{d})$ & $\beta$ & $-1.1(-1.4 ;-0.8)^{\mathrm{a}}$ & $-0.5(-0.8 ;-0.2)^{\mathrm{b}}$ & $-0.4(-0.7 ;-0.2)^{\mathrm{b}}$ \\
\hline
\end{tabular}

$\overline{\mathrm{a}, \mathrm{b}, \mathrm{c}}$ Values in the same row with unlike superscript letters are significantly different $(P<0.05)$.

The 35-day fish oil supplementation decreased AA content, and increased EPA content in erythrocytes both with time $(P<0.001)$ and in comparison with erythrocytes from pigs supplemented with sunflower oil and animal fat $(P<0.005)$. Plasma PGE 2 metabolite concentration decreased over time in pig's feed all three diets $(P<0.005)$, but the decrease was more distinct in fish oil supplemented pigs compared to the two other supplementations $(P<0.01)$.

Due to these results fish oil may have an immune modulating effect in this porcine model and further analysis of a broader range of inflammatory mediators are necessary to address this effect.

1. Calder PC (2004) Lipids 39, 1147-1161

2. Gao H, Lund L, Prag J et al. (2008) Eur J Vasc Endovasc Surg 35, 41-45. 